\title{
La percepción del alumnado de sexto grado de educación primaria hacia las personas que se encuentran en situación de discapacidad: la escala EPADI-1
}

\author{
Yonatan Díaz Santa María y Jesús Molina Saorín* \\ Facultad de Educación, Dpto. Didáctica y Organización Escolar, Universidad de Murcia, Campus Universitario 30100, \\ Murcia, España (correo-e: yonatan.diaz@um.es; jesusmol@um.es) \\ * Autor a quien debe ser dirigida la correspondencia.
}

Recibido May. 24, 2021; Aceptado Jul. 21, 2021; Versión final Sept. 15, 2021, Publicado Feb. 2022

\begin{abstract}
Resumen
El presente estudio valida el contenido y el cálculo de fiabilidad de la escala EPADI-1 (Escala de Percepción del Alumnado de Sexto de Educación Primaria hacia las Personas con Discapacidad). Se aplica la escala a un grupo de jueces expertos y a un grupo piloto. Las pruebas estadísticas incluyen el W de Kendall y el alfa de Cronbach. Los resultados destacan la visión que el propio alumnado mantiene de las personas con menor desempeño funcional, todo ello a través de una serie de ítems que han sido específicamente diseñados al respecto de sus experiencias escolares, y siempre dando observancia al Artículo 24 (derecho a la educación) de la Convención sobre los Derechos de las Personas con Discapacidad (CRPD, en inglés). Se concluye que la escala EPADI-1 tiene valores que conceden una excelente validez y fiabilidad del instrumento, quedando lista para su aplicación extensiva.
\end{abstract}

Palabras clave: percepción; diversidad funcional; educación primaria; política educativa

\section{Perception of sixth grade primary education pupils towards persons with disabilities: the EPADI-1 scale}

\begin{abstract}
The present study validates and calculates the reliability of the EPADI-1 scale (Student Perception Scale for 6th grade Primary Education towards Persons with Disabilities). The sampling scheme consists of a group of expert judges and a pilot group. The statistical analyzes performed include Kendall's W and Cronbach's alpha coefficient. The results highlight students' views about people with the lowest functional performance. This is conducted through a series of items specifically designed by considering both students' school experiences and Article 24 (the right to education) of the Convention on the Rights of Persons with Disabilities (CRPD). It is concluded that the EPADI-1 scale attained scores that ensure its validity and reliability, making it suitable for wide application.
\end{abstract}




\section{INTRODUCCIÓN}

No hay duda de que vivimos en una sociedad tan globalizada que aquello que pueda estar ocurriendo en un lugar específico del mundo (en una escuela concreta, por ejemplo) podría -con toda seguridad- ser un fiel reflejo de aquello que también está sucediendo en muchos otros lugares. Y es que la globalización ha permitido conocer la existencia de esas similitudes que los distintos territorios mantienen entre sí, independientemente de la región en la que se resida, de la cultura tan diferente que se pueda llegar a tener o del gobierno que lidere el país (Jared, 2020; Álvarez, et al., 2021). En este sentido, cabría destacar que la escuela de hoy -con independencia de su latitud-con toda seguridad se postula como uno de los agentes clave en la formación de los ciudadanos del mañana (Cook-Sather, 2020). Asimismo, si bien determinados eslóganes (como inclusión o calidad educativa) han acabado convirtiéndose en focos de atención prioritarios para esa sociedad globalizada, cabría preguntarse: ¿cuál es la realidad que el alumnado vive desde las aulas? Seguramente, este enfoque que tanto aboga por la diversidad e inclusión del alumnado, y aparentemente basado en los derechos, también participe de la construcción de un pensamiento organizado y estructurado alrededor de una escuela que premia -exclusivamente- a todos esos niños que son capaces de ajustarse al sistema escolar imperante, pero que -sin duda- continúa generando barreras para todos aquellos cuyo desempeño funcional es menor, o incluso para aquellos cuyas acciones son distintas a lo esperado con base a esa invisible norma social (Álvarez, et al., 2021).

Bajo estas premisas, cabría cuestionar si todavía hay alumnos que estén siendo castigados -segregados(en la escuela o en la sociedad) por motivo de su bajo desempeño funcional. Y es que -indudablementemientras se mantengan barreras hacia dichos alumnos se estará despreciando ese valor innegociable en virtud del cual habría de ser la escuela quien se ajuste al niño y no al revés, tal y como quedó debidamente promulgado por mor de la Convención Internacional de los Derechos de las Personas que se encuentran en situación de Discapacidad (CDPD, 2006) a través de su art. ${ }^{\circ} 24$ (dedicado al derecho a la educación), el cual señalaba -en su tenor literal- que los centros educativos deben realizar ajustes razonables en función de las necesidades individuales (p. 19).

Qué duda cabe que los distintos Estados Partes de dicha Convención fueron redefiniendo sus normativas educativas para -en principio- poder dar respuesta a la diversidad del alumnado; sin embargo, son múltiples los sociólogos y profesionales de la educación (Asorey y Martínez, 2017; Kart y Kart, 2021) que advierten que si bien las escuelas promueven escenarios integradores, estos no son -en absoluto- inclusivos, precisamente porque a pesar de que el alumnado con bajo desempeño funcional pueda estar presente en las aulas ordinarias, este no participa plenamente en las tareas o actividades junto al resto de sus compañeros (por ejemplo). Sobre esta base, la respuesta a las siguientes preguntas sería bastante predecible: ¿se sienten estos alumnos incluidos? ¿Siente el resto de los alumnos que esos niños con bajo desempeño funcional tienen los mismos derechos que ellos? Además, cuando abordamos el constructo inclusión escolar es necesario indicar que los distintos planteamientos aportados por la Administración educativa y consecuentemente- por los centros de enseñanza, están enmarcados en una visión reduccionista. Esto sucede porque sus acciones aun cuando pretenden trabajar en favor de la inclusión, se enfocan exclusivamente- desde la atención de la diferencia de este alumnado, concentrando sus esfuerzos sobre la posibilidad de supresión de esta diferencia, sin tener en cuenta que el objetivo debiera de ser promover escenarios en los que todos los niños puedan desarrollar al máximo sus capacidades sin tener que verse minusvalorados en virtud de un -tan caprichoso como consustancial al ser humano- desempeño funcional (Bastar, et al., 2021).

Y es que no se puede hablar de escuelas inclusivas por el simple hecho de que el alumnado cohabite en una misma aula o centro educativo, toda vez que no se garantizan las mismas posibilidades en tanto en cuanto no se ejecutan esos ajustes razonables de los que habla la legislación. De estar llevándose a cabo estos ajustes, con toda seguridad ningún niño se estaría quedando fuera del sistema educativo (Álvarez, et al., 2021; Díaz y Molina, 2021), como tampoco los niños con menor desempeño se sentirían desplazados durante las sesiones regulares de clase; buen ejemplo de ello es que -salvo honrosas excepciones- el desarrollo de la programación docente discurre desde una propuesta diseñada para un gran grupo (con alumnado promedio), existiendo -eso sí- toda una suerte de adaptaciones que suelen terminar en la realización de tareas completamente distintas (paralelas). Del mismo modo, tampoco tendrían por qué enfrentarse a la repetición de cursos con más frecuencia que el resto de sus compañeros para lograr los objetivos y estándares propuestos dando plena validez al aprendizaje homogeneizado (Álvarez, et al., 2021), que -por supuestodeprecia el valor que los niños confieren a sus aprendizajes, toda vez que sin un sentimiento de amor y pertenencia para con los compañeros y maestros, poco aprovechamiento de utilidad restará para el alumnado.

Sobre la base de estas consideraciones, se ha elaborado una escala por medio de la cual se pueda conocer la percepción que el alumnado finalista de educación primaria (concretamente de $6 .^{\circ}$ curso) mantiene al respecto de las personas con bajo desempeño funcional. La pretensión ha sido tratar de averiguar el grado 
de conocimientos y actitudes del alumnado participante con relación a las personas que se encuentran en situación de discapacidad, analizando si los alumnos con menor desempeño funcional continúan excluidos en el sistema educativo. Además, también se pretende conocer cuál es la visión de todos esos alumnos que nunca han tenido que enfrentarse a una serie de barreras sencillamente porque su desempeño funcional sí se ajusta a los criterios solicitados por una escuela eminentemente homogeneizadora. En ese sentido, resulta absolutamente necesario subrayar la importancia que tiene el hecho de dar voz al alumnado por considerarles agentes primordiales en los procesos educativos (Cook-Shater, 2020). Este hecho no solo resulta fundamental para poder transformar la escuela (Perfect y D'Amato, 2020; Abellán y Sáez, 2020), sino que sin tener pleno conocimiento sobre sus condicionantes y experiencias difícilmente podrá llegarse a conocer cuáles son sus necesidades $\mathrm{y}$, a partir de estas, potenciar contextos escolares que se aproximen a lo que los niños realmente requieren. Porque -como bien expresa la legislación educativa- el objetivo último es reforzar la equidad en la educación y la capacidad inclusiva del sistema (LOMLOE, 2020); por lo tanto, bajo esta óptica (y como proceso deductivo) ¿no sería lo más racional fomentar la participación de los niños durante el proceso de aprendizaje en lugar de continuar considerándoles como simples agentes pasivos?

La escala diseñada (nominada con el acrónimo EPADI-1), ha sido debidamente validada y estructurada alrededor de la definición de varios bloques temáticos: opinión personal sobre las personas que se encuentran en situación de discapacidad (aspectos generales); opinión personal sobre los derechos de las personas con bajo desempeño funcional; y opinión personal sobre las vivencias escolares de aquellos niños que se encuentran en situación de discriminación por bajo desempeño funcional. Como no podría ser de otro modo, los tres bloques se han elaborado de acuerdo a la literatura especializada en relación al estudio (Molina, 2017; Escobar, 2020; Díaz et al., 2021), siendo su finalidad indagar en la percepción que el alumnado mantiene alrededor de elementos tales como las creencias al respecto de las situaciones de discapacidad, su parecer sobre aspectos del modo de vida de las personas que se encuentran en situación de discapacidad, sus percepciones al respecto de lo que viven en primera persona (por tener un bajo desempeño funcional) o, en el caso de no ser así, sus creencias sobre lo que están viviendo sus compañeros con menor desempeño funcional (en función de lo que ven y comparten con ellos). Igualmente, y a modo de generalización y transferencia del conocimiento científico, la recogida de información aportada por la escala EPADI-1 permitirá -también- llevar a cabo un análisis comparativo con algunos de los campos incluídos en una escala previa (íntimamente relacionada, denominada EPREPADI-1) que tiene como participantes clave los maestros de educación primaria.

\section{OTROS ANTECEDENTES}

Indudablemente, la aproximación al término vulnerabilidad revela que se trata de un constructo de difícil definición en la medida que -históricamente- se ha venido estudiando desde muy diferentes perspectivas (Molina, 2017; Tabin, et al., 2021), tanto para la realización de estudios sociológicos o antropológicos, como también su uso se ha popularizado en la defensa del individualismo (es decir, en la protección de los derechos humanos). Por ello, en la medida que se ha ido ahondando en el término vulnerabilidad, también se ha logrado observar que la situación que comporta no es condición sine qua non de la persona (por su situación de salud, por ejemplo), sino que esta aparente fragilidad podría proceder de diversos escenarios (como bien pueden ser la ausencia de una estabilidad emocional, la falta de una continuidad laboral, la inestabilidad del núcleo familiar, etc.). De todo ello se ha logrado comprender que, generalmente, para que una persona sea denominada como vulnerable debiera haber sufrido un daño previo que le arrastraría a dicha situación (Illán y Molina, 2017; Díaz, et al., 2021). En este sentido, se puede confirmar que la vulnerabilidad se encuentra directamente relacionada con esa condición de normalidad que tanto ha marcado el transcurso histórico del ser humano, en tanto en cuanto los óbices para todas aquellas personas que se encuentren en situación de vulnerabilidad siempre serán los que socialmente se distancien de la conocida normalidad.

Evidentemente, el daño que pueden sufrir todas estas personas viene representado por la negación o dificultad de acceso a sus derechos, ya que es obligación tanto de la Administración como del conjunto de sujetos de derecho (en la medida que estos también tienen la obligación de proporcionar espacios tamizados por el diseño universal) comprometerse con la observancia de los mismos (Illán y Molina, 2017). Y es que mantener esa condición de normalidad supone favorecer la creación y conservación de espacios que representan tan solo a una parte de la sociedad, olvidándose (como viene ocurriendo) de los derechos de esos grupos considerados vulnerables y que infelizmente por desligarse de esa condición de normalidad, acaban siendo discriminados (Molina, 2017).

Resulta -igualmente- muy destacable señalar que siempre que bajo esa perspectiva de normalidad nos estemos refiriendo al ser humano, se estaría poniendo en valor la presunción de homogeneidad como principio fundamental en la representación de las sociedades. Y es que, a diferencia de lo que debiera estar sucediendo, socialmente se continúa poniendo el foco prioritario de atención en la dificultad que la persona puede llegar a tener para alcanzar un desarrollo personal pleno (lo que bien podría quedar definido por la 
consecución de una vida independiente), y no en cómo están planteados los diferentes espacios en los que deben desenvolverse (Doherty, 2020; Díaz, et al., 2021). Esto queda perfectamente definido por el concepto del diseño universal, el cual ha venido defendiendo -desde la década de 1960- que los espacios no deben ser rediseñados una vez que el proyecto ya ha finalizado, sino que debe tener presente (desde su diseño) a todas y cada una de las personas que pudieran llegar a hacer uso potencial de tales instalaciones, hecho que supondría una mayor eficiencia en el aprovechamiento de recursos tanto profesionales como económicos en lugar de diseñar para unos y, a la postre, acometer costosos cambios- (Yusof, 2020; Díaz, et al., 2021). Por todo ello, para este estudio que gira en torno a las personas que se encuentran en situación de discapacidad por motivo de su bajo desempeño funcional y, más concretamente, hacia la aproximación de esas situaciones de discriminación que el alumnado de educación primaria pueda estar viviendo, resulta absolutamente necesario destacar que determinadas conceptualizaciones como vulnerabilidad, homogeneidad o normalidad han venido definiendo el diseño y uso de los espacios regulares de aprendizaje.

Ciertamente, si bien en el terreno educativo se advierten enormes diferencias dependiendo -por ejemplo- del contexto geográfico al que hagamos referencia; sin embargo, si aludimos al escenario jurídico -en la mayoría de los casos- el derecho a la educación queda plenamente legitimado por el conjunto de la sociedad con independencia de las coordenadas geográficas (Molina, 2017). De modo análogo a la educación sucede con el concepto de calidad educativa, en la medida en que puede quedar ampliamente diferenciada dependiendo del territorio en el que se esté, de los recursos con los que se cuente, de la formación recibida por los maestros, de la cultura, etc (Álvarez, et al., 2021; Díaz, et al., 2021). Pero con independencia de ello, el reconocimiento del derecho a la educación (de calidad) es para todos los niños y con independencia del lugar en el que residan (Galiana, et al., 2021), siempre bajo esa mirada de igualdad de oportunidades (comprendiendo esta como dar a cada niño lo que realmente necesite al respecto de la consecución de su máximo desarrollo funcional $-\mathrm{y}$ no bajo ese principio de homogeneidad que pareciera haberse quedado arraigado a la propia cultura escolar, exponiendo una escuela denostada que no es capaz de ofrecer respuestas para la diversidad del alumnado-).

En el caso de España, la puesta en marcha de los ajustes razonables es obligatoria desde su incorporación tanto en la CDPD (art. ${ }^{\circ}$ 2) como desde la llegada de la Ley Orgánica 2/2006 de Educación (LOE), estableciendo a través de estas normativas que todas las escuelas -junto con la Administración- tendrán que dar una respuesta educativa al conjunto del alumnado independientemente del desempeño funcional de estos, creando espacios de aprendizaje para todos, y evitando -por supuesto- esa forma de separar a los niños por desempeño perdiendo de referencia que la educación tiene como uno de sus fines principales el desarrollo de la autonomía y crecimiento personal, sean cuales fueren las herramientas que haya que proporcionales.

Con la mirada puesta en la protección de los derechos, así como también en la posibilidad de ajustarse y ofrecer aquellas respuestas que -ciertamente- sean las que se adecuen a las necesidades de los niños (de acuerdo a sus deseos, aspiraciones y sueños -tal y como establece la normativa educativa-), surge el diseño universal para el aprendizaje (LOMLOE, 2020). Se trata de un movimiento originario del diseño universal arquitectónico y que -con posterioridad-se traslada al ámbito pedagógico con el fin de permitir explicar por qué hay alumnos que no logran ser incluidos en las aulas (en el sentido de estar plenamente considerados), aunque sí estén integrados (en el sentido de ubicados físicamente en el mismo espacio), en la medida en que su participación en el desarrollo habitual de las clases con el conjunto de sus compañeros generalmente es negada, justificando -además- una incapacidad del niño (Álvarez et al., 2021). Sin embargo, el transcurso del tiempo ha demostrado que lo que conocemos como discapacidad no es más que una situación que socialmente ha sido generada por barreras y actitudes, dando lugar -en consecuencia- a situaciones de discriminación (Perfect y D'Amato, 2020; Díaz, et al., 2021). Apréciese el valor de la incorporación -al constructo- del término situación, en la medida en que la eliminación o presencia de tales barreras y actitudes es -precisamente- el indicador para la supresión o aparición (respectivamente) de tales situaciones, concediendo así -la situación- una categoría de inestimable valor en la medida en que puede quedar bajo el control del ser (véase el legislador o la Administración -en interpretación de la ley-).

En el caso concreto de los alumnos con bajo desempeño funcional, el desempeño es considerado menor en tanto en cuanto los niños no sean capaces de ajustarse a esa situación de normalidad que la escuela ha generado a través de metodologías y políticas que son incapaces de abrirse a la diversidad funcional (Molina, 2017); es decir, si las políticas públicas mantuviesen la observancia por un currículo realmente flexible, los maestros no podrían cercenar las capacidades de sus alumnos (incluso las de aquellos que no tienen un bajo desempeño), y se dejaría atrás ese método libresco que da valor a las enseñanzas enlatadas, desconsiderando la valía del aprendizaje autónomo, del talento creativo, la crítica o la reflexión bajo la cual los maestros debieran ser algo muy sencillo (y complejo, a la vez): ser permanentes guías para su alumnado; nada más que eso, acompañándoles -en ocasiones de la mano- en todo su proceso de aprendizaje, ayudándoles a superar el conflicto cognitivo que la escuela prorrumpe sobre los niños (en contra de su natural tendencia entrópica), haciéndoles experimentar -desde el amor, la tolerancia y la comprensión- que la 
aventura del saber es terriblemente más emocionante cuanto más ignotos son los mares por los que se navega, siendo lo irrelevante que en esa misma travesía algunos vayan nadando, buceando, en catamarán o a hombros de gigantes (Álvarez, et al., 2021).

\section{MATERIALES Y MÉTODOS}

A continuación, se presenta el procedimiento que se ha seguido durante la elaboración de la escala EPADI1. Para ello, se explicará -en primer lugar- el diseño que se ha seguido para la construcción del instrumento, seguidamente se indica el método y la muestra que han dado forma a este proceso y, por último, se señalará cómo ha sido el proceso de validez y confiabilidad del contenido de la escala.

\section{Instrumento (escala EPADI-1)}

Inicialmente, es importante destacar que la escala EPADI-1 ha sido planteada para conocer la percepción que los estudiantes finalistas de educación primaria (específicamente de $6 .^{\circ}$ curso) mantienen al respecto de las personas que se encuentran en situación de discriminación por motivo de un bajo desempeño funcional. Para su diseño se han considerado diferentes variables implicadas en la configuración de las posibles creencias que el alumnado sostiene al respecto de las personas que se encuentran en situación de discapacidad (en general), como también aquellas percepciones que pudieran conservar en relación a aquello que observan y viven en sus escuelas (en particular) para aquella población. Precisamente, el instrumento se ha elaborado destacando algunos aspectos considerados esenciales, algunos de los cuales (a modo de ejemplo), destacamos a continuación. Por un lado, se somete a consideración si el alumnado que termina educación primaria mantiene estereotipos hacia aquellas personas que tienen un menor desempeño funcional; igualmente, se analiza en qué medida la escuela contribuye a la eliminación o conservación de tales estereotipos, así como si el alumnado sostiene que los niños con bajo desempeño funcional debieran tener derechos diferentes a los propios. En definitiva, a través de la escala EPADI-1 resultará posible definir si las escuelas están dando plena observancia al art. ${ }^{\circ} 24$ del derecho a la educación (recogido en la CDPD) o, por el contrario, la educación que el alumnado recibe continúa siendo excluyente.

En cuanto a su arquitectura, la escala ha sido diseñada de acuerdo a tres bloques temáticos (opinión personal al respecto de la discapacidad; opinión del alumnado sobre los derechos de las personas que se encuentran en situación de discapacidad; y opinión personal sobre sus vivencias o las de sus compañeros que se encuentran en situaciones de discriminación por motivo de un bajo desempeño funcional en la escuela). Por supuesto, cada uno de los bloques incluidos en la escala han sido definidos con base a la literatura especializada (Illán y Molina, 2017; Álvarez, et al., 2021; Díaz y Molina, 2021), y en comunión con el contenido de los diferentes artículos que dan conformidad a la CDPD. Se trata de un instrumento conformado por veintisiete ítems; los seis primeros están dedicados al conocimiento de los datos socio-demográficos (nombre del centro educativo en el que estudia, sexo, número de compañeros con bajo desempeño funcional...). Le siguen ocho preguntas - generales-sobre las situaciones de discapacidad, a través de las cuales se obtendrá tanto información de carácter cualitativo (para una de las cuestiones que contiene opciones de respuesta libre) como también cuantitativo (desde la base de una escala tipo Likert con cinco opciones de respuesta graduada de mayor a menor intensidad-). Por último, se incluyen doce ítems (también de tipo Likert) relacionados con las creencias acerca del reconocimiento de derechos de las personas con bajo desempeño funcional y sobre las propias vivencias del alumnado al respecto de dichas personas.

\section{Método y muestra}

Para llevar a cabo la validación del contenido de la escala EPADI-1, se ha seguido una doble vía (denominada modelo $U$ de validación). Por un lado, ha sido auditada por un grupo de jueces (expertos); y por otro (y con la finalidad de conceder mayor fiabilidad al instrumento), se ha aplicado a una muestra integrante de dos clases de $6^{\circ}$ de educación primaria.El método utilizado para la validación del instrumento ha seguido el procedimiento indicado por Campos (2020) referido tanto a la validez del contenido (con las contribuciones de los expertos), como también a la confiabilidad del instrumento (a través de la participación del grupo piloto). De acuerdo a la literatura especializada (Zamora, et. al., 2020; Sepúlveda et al., 2021), y al respecto del modelo U representado (Figura 1), se puede apreciar cómo la validación de la escala se ha desarrollado en dos períodos claramente diferenciados. Con objeto de conocer en qué medida los ítems propuestos debían ser modificados, en primer lugar se ha llevado a cabo una fase de validación de contenido (mediante juicio de expertos), en cuyo diseño se ha definido un instrumento de evaluación que ha sido utilizado por los jueces para su valoración. Esta rúbrica incorpora algunos datos y referencias sobre aspectos como la autoría del proyecto o el objetivo perseguido con el estudio, junto a unas breves instrucciones a tener en cuenta para acometer el proceso de evaluación (permitiendo -junto a la valoración de cada ítem- la formulación de propuestas de modificación). 
Con respecto a la valoración de los ítems, los criterios de inclusión utilizados (y debidamente definidos) han sido claridad, relevancia, coherencia y pertinencia, con una graduación en la puntuación extendida desde 1 a 4 (entendiendo 1 como el menor grado de acuerdo con el criterio valorado, y siendo 4 el mayor grado de acuerdo con el criterio puntuando). Del mismo modo, este instrumento de evaluación también ha ofrece la posibilidad de que los jueces expertos concedan una valoración general (para todo la escala) y que -si lo desean- incluyan unas observaciones generales. Finalmente, y una vez que se dio por concluida la fase de aplicación al grupo piloto, se realizaron los análisis de las valoraciones concedidas por los jueces expertos considerando como criterio para la conservación o supresión de los ítems la presencia de un grado de acuerdo de -al menos- el $75 \%$ de los jueces. Todo ello, de conformidad con lo establecido en el proceso de validación descrito por la literatura especializada (Álvarez, et al., 2021).

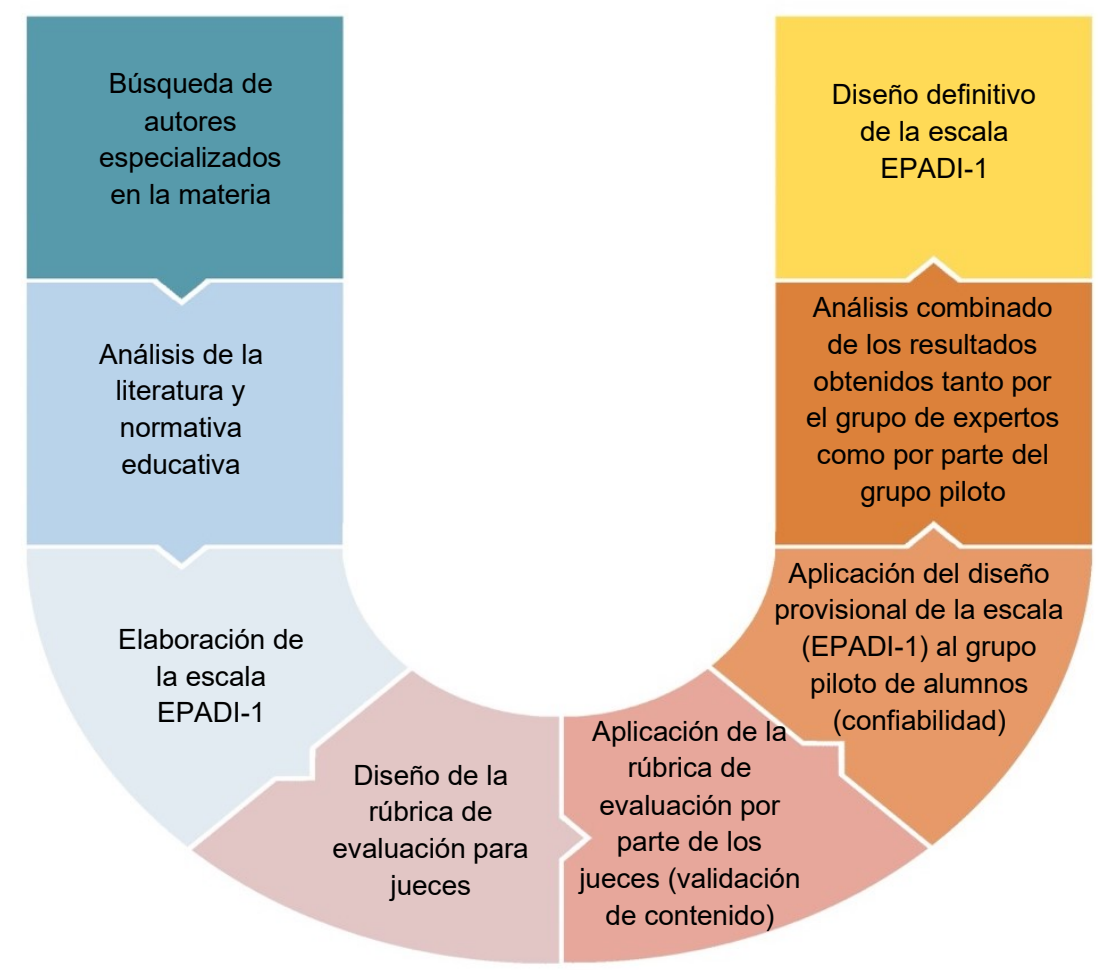

Fig. 1: Modelo U de validación: fases del procedimiento de validación de la escala EPADI-1.

\section{Validez y confiabilidad del instrumento}

Indudablemente, si bien existen diversos procesos para conocer la validez de contenido de un instrumento, concretamente para la escala EPADI-1 se realizó una selección de jueces de forma intencional; es decir, que la muestra fue -en todo caso- seleccionada atendiendo a la idoneidad del perfil profesional de los jueces (años de experiencia profesional, posesión de una categoría profesional vinculada con la educación -temática en base a la cual se construyó el instrumento-). De este modo, cabría señalar que el instrumento de evaluación se facilitó -para su cumplimentación, y a través de correo electrónico- a doce expertos que, atendiendo a su disponibilidad, tendrían que devolver su parecer en un período máximo de un mes con objeto de garantizar la máxima colaboración. Finalmente, la participación válida ascendió a un total de cinco jueces expertos $(41,6 \%$ de la totalidad de los jueces) y con una mortandad del $58,4 \%(n=7)$; entre los participantes finalistas se encontraban dos directores de centros de educación infantil y primaria (con cerca de veinte años de experiencia) y tres doctoras (titulares y catedráticas de universidad) con más de veinte años de experiencia dedicadas a la educación inclusiva.

En cuanto a la escala empleada para la valoración de los ítems por parte de los jueces, se construyó -tal y como ya se ha indicado- en base a una serie de criterios en relación a los cuales los jueces expertos tenían que expresar su grado de conformidad con los mismos. Los criterios de inclusión empleados -citados anteriormente- han sido los siguientes: claridad (referida a la comprensión semántica del ítem), relevancia (referida a la calidad de la información aportada por el ítem), coherencia (para conocer si el ítem es oportuno para la finalidad del estudio), y pertinencia (para saber si el ítem está bien redactado y si contribuye a alcanzar el objetivo del estudio). 
Posteriormente se llevó a cabo la fase dedicada a la prueba piloto, que supuso la aplicación de la escala EPADI-1 a dos grupos de $6 .^{\circ}$ de educación primaria (con un total de 140 participantes válidos). La pretensión de esta fase era conocer la confiabilidad del instrumento, en tanto en cuanto la conformidad expresada por los jueces expertos en todos los ítems implica -exclusivamente- la validez del contenido propuesto; sin embargo, por medio del análisis de los datos obtenidos del grupo piloto se puede conocer -también- si el instrumento mide ciertamente lo que se pretende, aportando a este análisis confiabilidad. En este sentido, para la validación del contenido se han calculado los estadísticos descriptivos resultantes de las valoraciones de los jueces para cada uno de los criterios expuestos. Además, se ha llevado a cabo (a través del programa Statiscal Product and Service Solutions, SPSS versión 24.0) la prueba $W$ de Kendall, estadístico no paramétrico, con la finalidad de conocer el grado de acuerdo entre los jueces para cada uno de los criterios evaluados. Igualmente, cabría mencionar que los valores resultantes de esta prueba oscilan entre 0 y 1 , siendo 1 el grado de mayor acuerdo y expresando 0 un grado de desacuerdo total (Brito et al., 2019; Campos, 2020).

Recuérdese que la escala EPADI-1 pretende conocer la percepción que el propio alumnado de $6 .^{\circ}$ de primaria mantiene al respecto de aquellas personas que se encuentran en situación de discriminación (por bajo desempeño funcional), tanto en sus entornos cotidianos (en general) como también en la escuela (en particular). En este sentido, para reducir posibles errores de la escala, junto a los procedimientos acometidos referidos a la fase de validación del contenido, también se ha realizado una prueba piloto, aplicando (en ambos procesos) la prueba no paramétrica de alfa de Cronbach. Este coeficiente se emplea -generalmente- para conocer la fiabilidad, y permite medir la correlación entre las variables que conforman una escala; además el alfa de Cronbach cuenta con diversas modalidades para conocer la fiabilidad de una escala (como puede ser la equivalencia entre test, la comparación entre un proceso de pretest y postest, y la consistencia interna de la escala, siendo este último el utilizado para analizar el grado en el que covarían los ítems entre sí).

\section{RESULTADOS Y DISCUSIÓN}

En primer lugar, y a partir de los datos recogidos en el proceso de validación de la escala EPADI-1, se han realizado los cálculos de los estadísticos descriptivos (media, desviación típica, valores máximo y mínimo, y cuartiles) para la totalidad de los criterios valorados por los jueces (claridad, relevancia, coherencia y pertinencia). Asimismo, se han considerado estos cálculos para las valoraciones realizadas por parte de los expertos al respecto del título, las instrucciones y su percepción general del cuestionario. Por otro lado, se ha llevado a cabo el cálculo del coeficiente de concordancia entre jueces - para cada uno de los criterios señalados- a partir de la prueba no paramétrica $W$ de Kendall (entendiendo que este coeficiente se comprende entre 0 y 1, cabría señalar que cuanto más se aproxime a 1 menor será la variabilidad entre las distintas valoraciones realizadas por los jueces expertos). Además, esta prueba permite conocer la consistencia interna entre jueces para la totalidad de los criterios de inclusión citados, y para ello se ha asumido como valor crítico $\alpha=0,05$. En segundo lugar, toda vez que se ha realizado el cálculo de los estadísticos descriptivos (Escobar y Cuervo, 2008; Campos, 2020), se han examinado las respuestas que obedecen a los valores mínimos 1 y 2 (al tratarse de un rango de conformidad bajo con el ítem valorado); y por otro lado, también se han observado aquellos ítems que sí se acercan a los valores 3 y 4 (al ser considerados el mayor grado de satisfacción interjueces).

A modo de ejemplo, en la siguiente tabla (Tabla 1 ) se exponen los estadísticos descriptivos de las respuestas otorgadas por los jueces para los criterios claridad $(C L)$, relevancia $(R)$, coherencia $(C H)$ y pertinencia $(P)$ relacionadas -exclusivamente- con aquellos ítems que han sido valorados con la puntuación mínima (entre 1 y 2), mostrando una desviación típica superior y, por tanto, confirmando una mayor variabilidad entre las evaluaciones de los jueces expertos. A la luz de los datos reflejados en la tabla mostrada (Tabla 1), se puede comprobar que la desviación típica con el índice más elevado y, por ende, con una mayor variabilidad de respuestas entre los jueces, aparece en el criterio relevancia (concretamente, en el ítem 6). Además, si se analiza el resto de criterios que aparecen en la tabla se puede observar cómo el ítem 6 muestra también una desviación típica elevada en los criterios de coherencia y pertinencia; esto confirmaría que este ítem ha sido evaluado (en un $25 \%$ ) por debajo de los niveles de acuerdo de los expertos. Lo mismo sucede con el ítem 12 , el cual ha sido valorado negativamente en los criterios de relevancia, coherencia y pertinencia también por un $25 \%$ de los jueces.

Del mismo modo, es destacable la valoración que los jueces han dado al ítem 21, ya que ha sido valorado en los criterios de claridad y coherencia con los índices mínimos (entre 1 y 2 ) por -al menos- el $25 \%$ de estos. Sin embargo, se puede asegurar que el índice de satisfacción ha sido elevado tanto para los ítems expuestos (valorados por el $75 \%$ de los jueces con puntuaciones de acuerdo máximo), como también para el resto de ítems (que han obtenido una valorización de acuerdo máximo -del $100 \%-$ ). La evaluación dada para los apartados título, instrucciones y diseño general de la escala, ha recibido un nivel de acuerdo elevado por el $100 \%$ de los jueces (con valores entre 3 y 4), lo que confirmaría el primer paso hacia la validez de dichos elementos. 
Del mismo modo, es destacable la valoración que los jueces han dado al ítem 21, ya que ha sido valorado en los criterios de claridad y coherencia con los índices mínimos (entre 1 y 2 ) por -al menos- el $25 \%$ de estos. Sin embargo, se puede asegurar que el índice de satisfacción ha sido elevado tanto para los ítems expuestos (valorados por el $75 \%$ de los jueces con puntuaciones de acuerdo máximo), como también para el resto de ítems (que han obtenido una valorización de acuerdo máximo -del 100 \%-).

Tabla 1: Estadísticos descriptivos de los ítems con mayor desviación típica.

\begin{tabular}{|l|l|l|r|r|r|r|r|r|r|}
\hline & \multicolumn{3}{|c|}{$N$} & & & & & \multicolumn{3}{|c|}{ Percentiles } \\
\cline { 10 - 12 } & Válidos & Perdidos & Media & Desv. típ. & Mínimo & Máximo & 25 & 50 & 75 \\
\hline CL7 & 5 & 0 & 3,40 &, 894 & 2 & 4 & 2,50 & 4,00 & 4,00 \\
\hline CL8 & 5 & 0 & 3,80 &, 447 & 3 & 4 & 3,50 & 4,00 & 4,00 \\
\hline CL9 & 5 & 0 & 3,20 & 1,095 & 2 & 4 & 2,00 & 4,00 & 4,00 \\
\hline CL15 & 5 & 0 & 3,40 &, 894 & 2 & 4 & 2,50 & 4,00 & 4,00 \\
\hline CL16 & 5 & 0 & 3,40 &, 894 & 2 & 4 & 2,50 & 4,00 & 4,00 \\
\hline CL19 & 5 & 0 & 3,40 &, 894 & 2 & 4 & 2,50 & 4,00 & 4,00 \\
\hline CL21 & 5 & 0 & 2,60 & 1,140 & 1 & 4 & 1,50 & 3,00 & 3,50 \\
\hline R6 & 5 & 0 & 3,00 & 1,414 & 1 & 4 & 1,50 & 4,00 & 4,00 \\
\hline R8 & 5 & 0 & 3,40 & 1,342 & 1 & 4 & 2,50 & 4,00 & 4,00 \\
\hline R12 & 5 & 0 & 3,20 & 1,095 & 2 & 4 & 2,00 & 4,00 & 4,00 \\
\hline CH6 & 5 & 0 & 3,40 &, 894 & 2 & 4 & 2,50 & 4,00 & 4,00 \\
\hline CH12 & 5 & 0 & 3,20 & 1,095 & 2 & 4 & 2,00 & 4,00 & 4,00 \\
\hline CH21 & 5 & 0 & 3,00 & 1,225 & 1 & 4 & 2,00 & 3,00 & 4,00 \\
\hline CH22 & 5 & 0 & 3,20 & 1,304 & 1 & 4 & 2,00 & 4,00 & 4,00 \\
\hline P6 & 5 & 0 & 3,20 & 1,095 & 2 & 4 & 2,00 & 4,00 & 4,00 \\
\hline P12 & 5 & 0 & 3,20 & 1,095 & 2 & 4 & 2,00 & 4,00 & 4,00 \\
\hline
\end{tabular}

La evaluación dada para los apartados título, instrucciones y diseño general de la escala, ha recibido un nivel de acuerdo elevado por el $100 \%$ de los jueces (con valores entre 3 y 4), lo que confirmaría el primer paso hacia la validez de dichos elementos. Del mismo modo, para conocer el grado de acuerdo entre los distintos jueces $(K=5)$ y considerando cada uno de los criterios valorados (claridad, relevancia, coherencia y pertinencia) al respecto de la totalidad de los ítems $(\mathrm{N}=27)$, se ha llevado a cabo la prueba no paramétrica $W$ de Kendall. Este estadístico ofrece la posibilidad de observar el grado de concordancia entre los jueces expertos, y sus valores resultantes siempre oscilan entre 0 y 1 (siendo 1 el acuerdo máximo y 0 un desacuerdo total). De este modo, a nivel general, el resultado obtenido en la prueba no paramétrica de W de Kendall para la totalidad de los criterios valorados por el conjunto de los expertos ha sido de 0,559. En este sentido, al obtener un acuerdo moderado entre los jueces, y con el objetivo de mejorar la validez de la escala, se ha llevado a cabo la prueba W de Kendall para cada uno de los criterios valorados por los jueces, pudiendo observar en qué criterios se obtiene una menor concordancia (en caso de no existir acuerdo entre los jueces se da la posibilidad de eliminar aquellos ítems que hayan obtenido una desviación típica mayor), pudiendo alcanzar un nivel de acuerdo por encima de 0,05 en el resultado de significación asintótica.

Tabla 2: Resultados de la prueba W de Kendall para los criterios de CL, RV, CH y PT.

\begin{tabular}{|c|c|}
\hline \multicolumn{2}{|c|}{ Kendall's coefficient of CL } \\
\hline $\mathrm{N}$ & 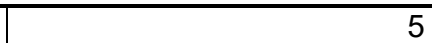 \\
\hline W de Kendall & ,307 \\
\hline Sig. asintótica &, 039 \\
\hline \multicolumn{2}{|c|}{ Kendall's coefficient of RV } \\
\hline $\mathrm{N}$ & 5 \\
\hline W de Kendall & ,230 \\
\hline Sig. asintótica & ,271 \\
\hline \multicolumn{2}{|c|}{ Kendall's coefficient of $\mathrm{CH}$} \\
\hline $\mathrm{N}$ & 5 \\
\hline W de Kendall & ,269 \\
\hline Sig. asintótica & 111 \\
\hline \multicolumn{2}{|c|}{ Kendall's coefficient of PT } \\
\hline $\mathrm{N}$ & 5 \\
\hline W de Kendall & 245 \\
\hline Sig. asintótica & ,199 \\
\hline
\end{tabular}


Como se puede comprobar a partir de los datos resultantes, el criterio de claridad no cumple con el valor mínimo establecido $(p=0,05)$, ya que su significación asintótica es de $p=0,039$ confirmando la existencia de diferencias estadísticamente significativas entre las valoraciones de los expertos. El resto de criterios analizados (RL, CH y PT) superan el valor mínimo y, por tanto, no cuentan con diferencias estadísticamente significativas. Asimismo, al no existir concordancia para el criterio calidad y tener un nivel mínimo de concordancia para el resto de los criterios (ya que los resultados están más próximos a 0 que a 1), resulta absolutamente necesario estudiar la posibilidad de eliminar aquellos ítems con menor valoración en la evaluación de los expertos. Una vez realizados los cálculos de los estadísticos descriptivos, así como también los que hacen referencia a los índices de concordancia entre los jueces, se ha procedido al cálculo de la consistencia interna de la escala EPADI-1 (Álvarez, et al., 2021). Para ello, se ha querido conocer los intervalos de confianza aportados por la prueba alfa de Cronbach; esta se ha llevado a cabo teniendo en cuenta la totalidad de los ítems $(K=27)$, así como también se ha considerado para su interpretación que generalmente- a partir de 0,7 la fiabilidad de la escala es aceptable. En el caso de la EPADI-1, el resultado ha sido $\alpha=0,943$; siguiendo los criterios de George y Mallery (2003), este dato señala una consistencia interna excelente, afirmando una alta correlación entre los ítems y las puntuaciones concedidas por los jueces. Esto -a su vez- estaría ratificando una adecuada redacción de los ítems, así como que estos realmente miden lo que se desea.

Tabla 3: Coeficiente alfa de Cronbach para el grupo de jueces expertos.

\begin{tabular}{|c|c|}
\hline \multicolumn{2}{|c|}{ Estadísticas de fiabilidad } \\
\hline Alfa de Cronbach & $\mathrm{N}$ de elementos \\
\hline, 943 & 120 \\
\hline
\end{tabular}

Si bien es cierto que la validación del contenido -por parte de los jueces expertos- ya confiere fiabilidad al instrumento (en tanto en cuanto se lleva a cabo un proceso completo de análisis de fiabilidad para la escala que se desea validar), se decidió realizar una prueba piloto que fuese capaz de conceder mayor grado de excelencia al proceso de validez. En este sentido, se ha calculado el coeficiente de alfa de Cronbach para el grupo piloto (140 alumnos). Además, siguiendo los criterios de George y Mallery (2003) se puede confirmar la valoración buena del instrumento al obtener un resultado superior a $0,7(\alpha=0,752)$.

Tabla 4: Resultados de la prueba alfa de Cronbach para el grupo piloto.

\begin{tabular}{|c|c|}
\hline \multicolumn{2}{|c|}{ Estadísticas de fiabilidad } \\
\hline Alfa de Cronbach & $\mathrm{N}$ de elementos \\
\hline, 752 & 27 \\
\hline
\end{tabular}

Por otro lado, con los datos recogidos del grupo piloto, se ha llevado a cabo un análisis factorial exploratorio que tiene por objeto la comprobación de la fiabilidad y confiabilidad de la escala. En este sentido, los nuevos aportes resultantes tras la realización del análisis factorial exploratorio (AFE), permitirán mostrar la relación que tienen unos ítems con otros. Cabe señalar, que se han reportado un total de 7 factores que podrán explicar la covarianza entre las variables. Concretamente, en la tabla (Tabla 5) se detalla cómo estos factores explican el 66,92 \% del total de la varianza. Asimismo, para realizar dicho análisis, se señalaron una serie de condicionantes iniciales como fueron la prueba Kaiser-Meyer-Olkin (KMO) y la prueba de esfericidad de Barlett. Para el primer caso, el resultado fue de 0,713, esto confirma una relación entre las variables satisfactoria, así como también define que unos ítems pueden ser explicados por otros (Álvarez, et al., 2021); para el segundo caso, los resultados explican la presencia de igualdad entre las varianzas (también definida como homocedasticidad), al obtener un valor igual a cero.

Tabla 5: Matriz de la varianza total explicada para los factores resultantes.

\begin{tabular}{|c|c|c|c|}
\hline \multirow{2}{*}{ Factores } & \multicolumn{3}{|c|}{ Autovalores iniciales } \\
\cline { 2 - 4 } & Total & \% de varianza & $\%$ acumulado \\
\hline 1 & 3,294 & 18,299 & 18,299 \\
\hline 2 & 2,328 & 12,933 & 31,232 \\
\hline 3 & 1,602 & 8,898 & 40,130 \\
\hline 4 & 1,345 & 7,473 & 47,603 \\
\hline 5 & 1,286 & 7,144 & 54,748 \\
\hline 6 & 1,115 & 6,195 & 60,943 \\
\hline 7 & 1,078 & 5,986 & 66,929 \\
\hline
\end{tabular}


A la vista de los datos analizados, toda vez que los resultados todavía no permiten confirmar la consistencia interna de la escala, así como tampoco certificar su validez del contenido (en tanto en cuanto para el criterio claridad los jueces no han llegado a un nivel de acuerdo satisfactorio y para el resto de los criterios relevancia, coherencia y pertinencia- el análisis arroja unos resultados de acuerdo bajos, debido a su mayor proximidad al valor 0), con el objetivo de obtener unos resultados de validez del contenido altamente satisfactorios, se hizo necesario reestructurar la escala EPADI-1 en aplicación de los cambios planteados por los jueces. Sobre esta base, se eliminaron aquellos ítems que habían obtenido una desviación típica superior a 1 y que, por ende, habían sido evaluados por los jueces -en alguno de los criterios- con las puntuaciones mínimas (entre 1 y 2). Por otro lado, se realizaron las modificaciones de los ítems que habían sido valorados positivamente por los jueces pero que -a su vez- habían recibido algún tipo de observación por parte de estos (debidamente indicadas en el apartado de observaciones que se incluía para cada uno de los ítems). En este sentido, a continuación se presentan aquellos ítems que han sido eliminados de la escala, así como también la propuesta de modificación sobre algunos ítems por parte de los expertos.

Teniendo en consideración las modificaciones expuestas en la tabla mostrada (Tabla 6), cabría resaltar los datos resultantes toda vez que los análisis estadísticos se volvieron a realizar para conocer -nuevamente- la validez del contenido y la fiabilidad de la escala. En este sentido, se presentan -a continuación- los datos correspondientes a la prueba $W$ de Kendall (que fue realizada para la totalidad de los criterios valorados por los jueces). Tal y como se puede observar (Tabla 7), a diferencia de los primeros datos extraídos (expuestos en la tabla 2), todos los criterios superan el valor mínimo $(p=0.05)$ y si bien existe dispersión entre los valores obtenidos, este hecho quedaría perfectamente justificado al tratarse de resultados más probables y usuales que la propia aproximación a los valores extremos de 0 y 1 (Escobar y Cuervo, 2008). Igualmente, se pone de manifiesto que el criterio de claridad ahora cuenta con un nivel de acuerdo entre los jueces perfectamente aceptable (superando el mínimo), al tiempo que también se observa cómo el resto de criterios han pasado de tener un índice de acuerdo algo ambiguo (inicialmente) a un nivel moderado-alto (en la actualidad).

Tabla 6: Propuesta para la eliminación o modificación de ítems.

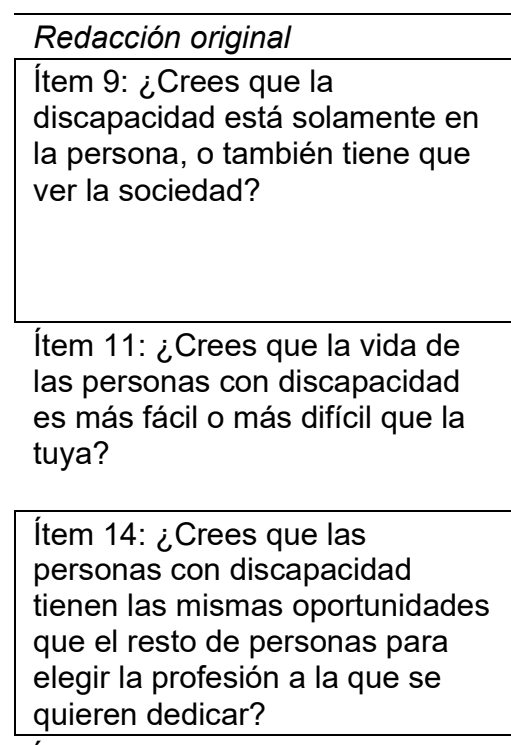

Ítems 16: ¿Crees que los adultos con discapacidad deberían tener derecho a vivir solos (como el resto de adultos)?

Propuesta de modificación

Propuesta del experto (E3): Revisar el enunciado, la segunda parte no queda claro. En vez de "ver" se podría sustituir por "influye".

Propuesta del experto (E5): Quizá la pregunta se debería formular de manera más directa.

Propuesta del experto (E5): Se presupone que no tiene discapacidad quien contesta; el ítem quedaría mejor redactado del siguiente modo “¿Cómo crees que es la vida de las personas con discapacidad?

Propuesta del experto (E5): Se podría eliminar "a la que se quieren dedicar"

Propuesta del experto (E3): Sustituir "solos" por "independientes" Propuesta del experto (E4): Sustituir el inicio por: ¿Crees que las personas adultas...

Ítems 18: ¿Piensas que los adultos con discapacidad deberían tener derecho a tener hijos?

Ítems 24: Creo que a los niños con discapacidad solo deberían darles clase los maestros de educación especial.
Propuesta del experto (E2): Escribiría "las personas adultas" en lugar de "los adultos".

Propuesta del experto (E4): Sustituir completamente por "creo que a los niños con discapacidad solo deberían darles clase los maestros de educación especial en un aula especial"

\section{Redacción definitiva}

Ítem definitivo: ¿Crees que la discapacidad está solamente en la persona, o también influye la sociedad?

Ítem definitivo: ¿Cómo crees que es la vida de las personas con discapacidad?

Ítem definitivo: ¿Crees que las personas con discapacidad tienen las mismas oportunidades que el resto de personas para elegir su profesión?

Ítem definitivo: ¿Crees que las personas adultas con discapacidad deberían tener derecho a vivir independientes (como el resto de adultos)?

Ítem definitivo: ¿Piensas que las personas adultas con discapacidad deberían tener derecho a tener hijos?

Ítem definitivo: Creo que a los niños con discapacidad solo deberían darles clase los maestros de educación especial en un aula especial.

Se han eliminado de la escala los ítems 6, 12 y 21 por presentar una desviación típica superior a 1 en (al menos) tres de los criterios valorados por los expertos. 
Tabla 7: Resultados de la prueba W de Kendall una vez realizada la propuesta de modificaciones.

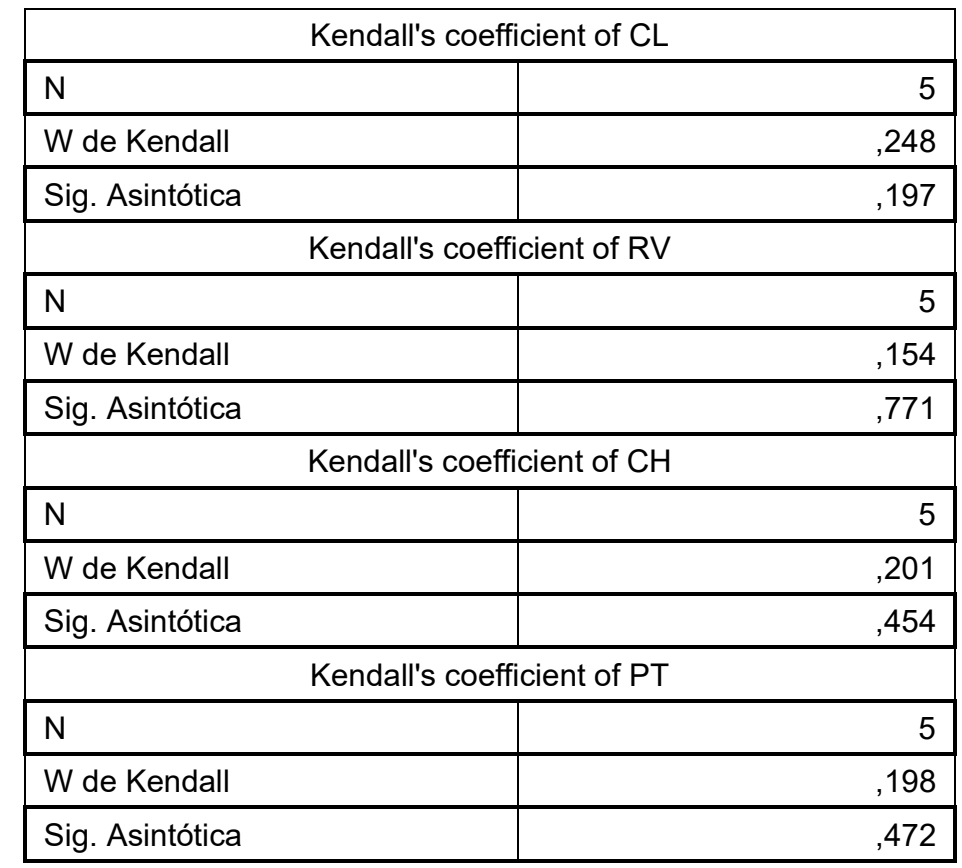

A su vez, se ha vuelto a realizar la prueba del coeficiente alfa de Cronbach, pero en esta ocasión sin los ítems suprimidos $(K=24)$, dando como resultado una variación insignificante $(\alpha=0,936)$, hecho que redunda en destacar una consistencia interna excelente para toda la escala. En este sentido, se puede confirmar que, una vez realizadas las modificaciones sugeridas por los jueces expertos, así como la propuesta de supresión de ítems de acuerdo a los datos extraídos del primer análisis estadístico, la escala EPADI-1 muestra una validez de contenido y una fiabilidad sobresaliente.

\section{DISCUSIÓN}

Con toda seguridad, si echáramos la vista atrás podríamos comprobar que -al menos en apariencia- el escenario educativo en el que han aprendido los niños con menor desempeño funcional ha experimentado grandes modificaciones con el pasar de los años (Molina, 2017; Erstad, et al. 2021); sin embargo, estudios como el aquí presentado facilitarán la obtención de datos precisos al respecto del alcance y naturaleza de tales cambios, en la medida en que no solo permitirán corroborar la existencia de situaciones de discriminación en las escuelas, sino también conocer cómo se ha evolucionado y en qué aspectos se debe seguir poniendo el foco prioritario de atención (por parte de los maestros y de la Administración) en pro de alcanzar ese deseado modelo de los derechos humanos, en virtud del cual todos los niños deban crecer sin ser desconsiderados, discriminados o apartados bajo esa falsa creencia de que solo hay un camino para enseñar.

El anuncio de exclusión social que amenaza a quienes tienen un bajo desempeño funcional siempre ha sido una problemática de carácter estructural, ya que -generalmente- cuando se aborda el constructo de la exclusión son múltiples las esferas sociales que comprometen la vida de las personas más vulnerables. En este sentido, tras la lectura de la literatura especializada (Tobias y Mukhopadhyay, 2017; Valentim, 2020) se ha podido observar que las personas que tienen bajo desempeño funcional -todavía hoy- se encuentran viviendo numerosas situaciones de discriminación que -de no hacer nada por remediarlo- les condenarán a una irremediable exclusión social.

Concretamente, y dentro del panorama educativo actual, son numerosas las ocasiones en las que el alumnado (en contra de lo que establece la ley) es discriminado tomando como ratio decidendi el bajo desempeño funcional, denominando genéricamente a esa situación de discriminación (por motivo de desempeño funcional) como situación de discapacidad o situación de discriminación por motivo de desempeño funcional. Superada la perplejidad (y el bochorno) inicial que pueda producirnos el conocimiento de la continuidad de tal situación (toda vez que se tenga vasto conocimiento del tenor literal de la legislación), bien cabría afirmar que esta generación de situaciones de discriminación ( $\mathrm{y}$, por ende, de violación del derecho constitucional de los menores) es amparada por una Administración que no tiene entre sus prioridades la plena observancia del derecho a la educación en igualdad de oportunidades (Molina, 2017; Álvarez, 2021); como también cabría sospechar que los maestros ni conocen la totalidad de medidas ordinarias de atención a la diversidad contempladas en la legislación, ni sostienen la necesaria reflexión crítica 
que les concitase la necesidad de adecuar los currículos para que -tal y como establece la ley educativa en su preámbulo- en ningún caso existan barreras infranqueables para el alumnado (por ejemplo, aplicando el diseño universal de aprendizaje).

Además, los primeros resultados obtenidos tras la aplicación de la escala al grupo piloto, y tras la realización del análisis factorial exploratorio, señalan un vínculo entre el ítem que hace referencia a la creencia de la igualdad de derechos para todos los niños y el hecho de que aquellos con menor desempeño funcional tengan que ir a un centro de educación especial. Específicamente, existe un alto porcentaje de alumnos que piensan que los niños que se encuentran en situación de discapacidad debieran tener los mismos derechos que el resto, pero sin embargo consideran que los centros de educación especial son el mejor destino para estos niños que tienen un bajo desempeño en las escuelas. Así como también opinan -en su inmensa mayoríaque únicamente los maestros de educación especial tendrían que ser los encargados de la educación de estos alumnos. Del mismo modo, se percibe un vínculo entre las cuestiones que hacen referencia al hecho de que la enseñanza es más sencilla para el alumnado que se encuentra en situación de discapacidad y la percepción de que les guste ir a la escuela o se sientan tristes en esta. Particularmente, una gran mayoría manifiesta que no están seguros (o no están de acuerdo) con el hecho de que los niños con bajo desempeño funcional lo tengan más fácil que el resto de alumnos en la escuela (porque sus exámenes sean más fáciles y tengan menos deberes), pero -al mismo tiempo-consideran que a estos alumnos no les gusta ir a la escuela y que además se sienten tristes en ella.

Precisamente para desvendar este misterio, con los resultados obtenidos a partir de la aplicación generalizada de la escala EPADI-1 se pretende iniciar un debate colectivo (familias, Administración y centros educativos) con el que dar a conocer la situación actual en la que se encuentran los alumnos que -en la escuela- muestran bajo desempeño funcional, así como dar voz al alumnado al respecto de lo que conocen (o desconocen) sobre las personas que se encuentran en situación de discriminación por motivo de bajo desempeño funcional (en general), analizando su percepción sobre la prevalencia de estereotipos en la escuela (en particular). Del mismo modo, los resultados permitirán conocer si las actuaciones generadas por la Administración y los maestros desde la entrada en vigor de la CDPD está resultando provechosa para el alumnado, si se están reduciendo las situaciones de discriminación en la escuela o si, por el contrario, este escenario continúa marcado por los prejuicios y la exclusión del alumnado con menor desempeño funcional. Por lo tanto, con base a todo lo expuesto y una vez alcanzado el diseño definitivo de la escala, se puede confirmar que este estudio de validación muestra unos resultados finales que ratifican la validez y fiabilidad para la aplicación masiva de la EPADI-1 en tanto en cuanto los resultados psicométricos desprendidos de las pruebas aplicadas arrojan unos índices satisfactorios en relación al propósito de la investigación.

\section{CONCLUSIONES}

De acuerdo al trabajo presentado y a los resultados obtenidos, se pueden plantear las siguientes conclusiones principales:

1.- La realización del juicio de expertos ha facilitado la evaluación del contenido a través de los criterios de análisis, destacando la claridad de la semántica de los ítems, la relevancia que se le otorga a la calidad de la información tratada en los ítems, la coherencia en relación a la adecuación y finalidad para cada uno de los ítems de acuerdo al objetivo del estudio y la pertinencia en relación a la redacción y contribución de los ítems a la finalidad del estudio. Todo ello, permite -junto con las técnicas de análisis de datos- evaluar el diseño inicial de la escala EPADI-1.

2.- Del mismo modo es destacable para la validación del contenido, el proceso que se ha seguido en la selección del perfil del experto, así como también el diseño de un instrumento que se ha concretado en el uso de criterios y coeficientes que han facilitado la claridad durante el proceso del juicio de expertos y que -a su vez- han sido del todo necesarios para conceder unos resultados determinantes.

3.- Igualmente, el resultado de $W$ de Kendall demuestra que las puntuaciones otorgadas por los expertos con relación a los criterios evaluados, conceden un nivel de concordancia satisfactorio.

4.- La realización de una prueba piloto ha permitido dar mayor consistencia al proceso de validación de la escala, concluyendo unos resultados sobresalientes para las pruebas realizadas de KMO y de esfericidad de Barlett, así como también se destaca la existencia de siete factores determinantes que resultan de la realización del análisis factorial exploratorio y que explican un alto porcentaje de la varianza del instrumento.

Por todo ello, las pruebas realizadas han permitido llevar a cabo una reestructuración del cuestionario que, sin lugar a dudas, y tras las modificaciones expuestas ut supra, se puede constatar que el contenido de la escala es el adecuado para el propósito de la investigación por lo que -superada esta fase de validaciónprocede la aplicación extensiva de la EPADI- 1 al alumnado de $6 .^{\circ}$ de educación primaria. 


\section{REFERENCIAS}

Abellán, J., y Sáez-Gallego, N., Opiniones relativas a la inclusión de los alumnos con necesidades educativas especiales mostradas por futuros maestros de infantil y primaria, doi:doi.org/10.5209/rced.62090, Revista Complutense De Educación, 31(2), 209-229 (2020)

Alisa, K., Hollingshead, A., y Howery, K., A Closer Look: Examining Teachers' Language Around UDL, Inclusive Classrooms, and Intellectual Disability, doi:10.1352/1934-9556-55.1.15., Intellectual and Developmental Disabilities, 55(1), 15-24 (2017)

Álvarez, J.M., Díaz, Y., y Molina, J., El Código Cuomo. Las fábulas de María: una niña a la que no le gustaba la escuela, Dykinson, Madrid, España (2021)

Álvarez, J. M., Molina, J., Trigueros, F. J., y Miralles, P., The Development of Historical Competencies in Secondary Education: A Study Based on the Analysis of Sources in Spanish and Italian History Textbooks, doi: doi.org/10.26803/ijlter.20.4.8, International Journal of Learning, Teaching and Educational Research, 20(4), 137-151 (2021)

Asorey, M. J. F., y Martínez, S. S., Creando y adaptando materiales para compartir: Una experiencia con futuros maestros, doi:doi.org/10.6018Zj/308951, Educatio Siglo XXI, 35(3), 153-174 (2017)

Bastart, J., Rohmer, O., y Popa-Roch, M., Legitimating Discrimination against Students with Disability in School: The Role of Justifications of Discriminatory Behavior, doi:doi.org/10.5334/irsp.357, International Review of Social Psychology, 34(1) (2021)

Brito, M., López, J., y Tobón, S., Rubric Validation for the Review of Didactic Planning in Initial Teaching Training, doi:doi.org/10.1166/asl.2019.13183, Advanced Science Letters, 25(1), 47-49 (2019)

Campos, M.L., Velasco, C.B., y Araya, J.P., Adaptación y validación de escalas de medición en el trabajo. Parte 2: eficacia colectiva, doi:dx.doi.org/10.4067/S0718-07642020000600043, Información Tecnológica, 31(6), 43-52 (2020)

Cook-Sather, A., Student voice across contexts: Fostering student agency in today's schools, doi:doi.org/10.1080/00405841.2019.1705091, Theory into Practice, 59(2), 182-191 (2020)

Díaz, Y., y Molina, J., Persons with disabilities facing the mirage of the right to education: a systematic review of the literatura, doi:doi.org/10.3389/feduc.2021.706372, Frontiers in Education, 6, 1-9 (2021)

Doherty, G., Prejudice, friendship and the abuse of disabled people: An exploration into the concept of exploitative familiarity ('mate crime'), doi:doi.org/10.1080/09687599.2019.1688646, Disability \& Society, 35(9), 1457-1482 (2020)

Erstad, O., Miño, R., y Rivera-Vargas, P., Educational practices to transform and connect schools and communities, doi:doi.org/10.3916/C66-2021-01, Comunicar, 29(66), 9-19 (2021)

Escobar, M., Muñoz, D., Piñones, C., y Cuadra, D., Tiempo escolar e inclusión educativa: un estudio de teorías subjetivas de profesores, http://dx.doi.org/10.4067/S0718-07642020000500139, Información Tecnológica, 31(5),139-151 (2020)

Escobar, J., y Cuervo, Á., Validez de contenido y juicio de expertos: una aproximación a su utilización, Avances en Medición, 6(1), 27-36 (2008)

Galiana, M. M., Vicente, M. Á. G., y Martínez, A. C. Creencias de los estudiantes de educación secundaria obligatoria sobre la diversidad funcional, doi:doi.org/10.6018/educatio.469331, Educatio Siglo XXI, 39(1), 349-373 (2021)

George, D., y Mallery, P., SPSS for Windows step by step: A simple guide and reference, 11.0 update. Boston: Allyn \& Bacon (2003)

Illán, N., y Molina, J., El artículo 8 de la CDPD -toma de conciencia-. Visiones y perspectivas desde el colectivo de personas con diversidad funcional intelectual, Revista de Educación Inclusiva, 6(1), 59-74 (2017)

Jared, F., Liberal individualism and the globalization of education as a human right: The worldwide decline of early tracking, 1960-2010: A magazine of theory and practice, doi:doi.org/10.1177/0038040719873848, Sociology of Education, 93(1), 1-19 (2020)

Kart, A., y Kart, M., Academic and social effects of inclusion on students without disabilities: A review of the literature, doi:doi.org/10.3390/educsci11010016, Education Sciences, 11(1), 2-13 (2021)

Molina, J., La discapacidad empieza en tu mirada. Las situaciones de discriminación por motivo de diversidad funcional: escenario jurídico, social y educativo, Madrid: Delta Publicaciones (2017)

ONU, Convención sobre los Derechos de las Personas con Discapacidad y Protocolo Facultativo. Nueva York: ONU (2006)

Perfect, M. M., y D'Amato, R. C., Introduction to special issue on the history of the future of proactive school psychology: Transcending the past, excelling in the present, and transforming the future, doi:doi.org/10.1037/spq0000419, School Psychology, 35(6), 363 (2020)

Sepúlveda, J. J., Gálvez, F. A., Vilches, C., y Hinojosa, H., Variables relevantes para el escepticismo profesional en la formación de contadores auditores, doi:doi.org/10.4067/S0718-07642021000400099, Información Tecnológica, 32(4), 99-108 (2021) 
Tabin, M., Diacquenod, C., y otros cuatro autores, Cross-cultural preliminary validation of a measure of social vulnerability in people with intellectual disabilities, doi:doi.org/10.3109/13668250.2020.1793450, Journal of Intellectual \& Developmental Disability, 46(1), 67-79 (2021)

Tobias, E., y Mukhopadhyay, S., Disability and social exclusion: Experiences of individuals with visual impairments in the Oshikoto and Oshana regions of Namibia, doi:doi.org/10.1177/0971333616689203, Psychology and Developing Societies, 29(1), 22-43 (2017)

Valentim, A., y Joaquim, P. V., What I think of school: perceptions of school by people with intellectual disabilities, doi:doi.org/10.1080/09687599.2019.1702507, Disability \& Society, 35(10), 1618-1640 (2020)

Yusof, Y., Cheong, C. C., y otros tres autores, Improving inclusion of students with disabilities in Malaysian higher education, doi:doi.org/10.1080/09687599.2019.1667304, Disability \& Society, 35(7), 1145-1170 (2020)

Zamora, M. S., Serrano, F. J., y Martínez, M., Validez de contenido del modelo didáctico P-VIRC (preguntar-ver, interpretar, recorrer, contar) mediante el juicio de expertos, doi:doi.org/10.4067/S0718-50062020000300043, Formación Universitaria, 13(3), 43-54 (2020) 